\title{
Datos epidemiológicos de lesiones en accidentes de tránsito. Encuesta del período enero 2017-julio 2020 Comité de Morbimortalidad - AAOT
}

\author{
Débora Gamarra," Mónica N. Sierto,," Germán Garabano,, Adriana Cubecino,"\#" Nicolás A. Robador, $\$$ \\ Harold Simesen de Bielke, ${ }^{\S \S}$ Javier Olivetto ${ }^{\dagger}$ \\ *Casa de Auxilio de Ramos Mejía, Buenos Aires, Argentina \\ **Hospital Interzonal General de Agudos "Dr. Rodolfo Rossi”, La Plata, Buenos Aires, Argentina \\ ${ }^{\#}$ Hospital Británico, Ciudad Autónoma de Buenos Aires, Argentina \\ \#Clínica Chapelco - CMIC, San Martín de los Andes, Neuquén, Argentina \\ ${ }^{\S}$ Hospital Regional “Dr. Enrique Vera Barros”, La Rioja, Argentina \\ §sanatorio Modelo de San Miguel de Tucumán, Tucumán, Argentina \\ ${ }^{\ddagger}$ Sanatorio Americano de Rosario, Santa Fe, Argentina
}

\section{RESUMEN}

El aumento progresivo y constante de las muertes y la discapacidad por accidentes de tránsito es, en la actualidad, reconocida como un problema epidémico de salud pública nacional y global. La Organización Mundial de la Salud y múltiples organismos trabajan mancomunadamente para consolidar un registro sistemático de los siniestros viales y sus consecuencias en términos de morbimortalidad. Se busca generar información que contribuya a la toma de decisiones en la gestión y la coordinación de estrategias para el abordaje de esta problemática. La Asociación Argentina de Ortopedia y Traumatología, a través de la creación del Comité de Morbimortalidad hace ya una década, ha impulsado la generación de un registro nacional de lesiones traumatológicas. Este segundo informe sobre las encuestas acumuladas desde 2017 hasta julio de 2020 tiene como objetivo general presentar los datos recopilados sobre las lesiones traumatológicas provocadas por accidentes de tránsito. El objetivo específico es promover la incorporación de estas encuestas en nuestros Servicios como un instrumento de registro. Convocamos a renovar el compromiso de todas las instituciones del país, nucleadas en esta Asociación, en la tarea de recopilar datos epidemiológicos que finalmente nos permitan mejorar la validez científica de nuestra práctica y, por ende, de las publicaciones de nuestra especialidad.

Palabras clave: Lesiones; accidentes de tránsito; siniestros viales.

Nivel de Evidencia: II

Epidemiological Data on Injuries in Road Traffic Accidents. Surveys From the Period January 2017 to July 2020. Morbidity and Mortality Committee - AAOT

\section{ABSTRACT}

The constant and progressive increase in mortality and disability caused by road traffic accidents is currently recognized as an endemic problem of Public Health in the national and global levels. WHO and multiple organizations work together to achieve a systematic record of road accidents and their consequences in terms of morbidity and mortality. The purpose is to generate information that contributes to decision-making in management and coordination of strategies to address this problem. Through the creation of the CMM (Morbidity and Mortality Committee) a decade ago, the AAOT has promoted the generation of a national registry of trauma injuries. This second report (based on the surveys accumulated from 2017 to July 2020) has as a general objective to describe the data collected in relation to trauma injuries caused by traffic accidents. The specific objective is to promote the incorporation of these surveys in our services, as a registration instrument. We call to renew the commitment of all institutions nucleated in this Association at the national level in the task of collecting epidemiological data that finally will allow us to improve the scientific validity of our practice and, therefore, of the publications of our specialty.

Key words: Injuries on traffic accidents; injuries in road accidents.

Level of Evidence: II

Recibido el 27-2-2021. Aceptado luego de la evaluación el 12-3-2021 • Dra. DÉBORA GAMARRA • doctora.debora@ gmail.com

https://orcid.org/0000-0001-7661-850X

Cómo citar este artículo: Gamarra D, Sierto MN, Garabano G, Cubecino A, Robador NA, Simesen de Bielke H, Olivetto J. Datos epidemiológicos de lesiones en accidentes de tránsito.

Encuesta del período enero 2017-julio 2020. Comité de Morbimortalidad - AAOT. Rev Asoc Argent Ortop Traumatol 2021;86(6):829-841. https://doi.org/10.15417/issn.1852-7434.2021.86.6.1326 


\section{INTRODUCCIÓN}

Desde hace ya muchos años, la Organización Mundial de la Salud alerta sobre el avance de "la epidemia mundial de las enfermedades y muertes causadas por siniestros viales" más comúnmente denominados "accidentes de tránsito". Es permanente el aumento de víctimas por esta causa en el mundo.

Según la Organización Mundial de la Salud y las fuentes estadísticas sanitarias mundiales, la muerte por traumatismos ocasionados en accidentes de tránsito pasará de ser la novena causa de muerte a la quinta para 2030. Las estadísticas de 2004 ya revelaron más de 1,2 millones de muertes anuales, en el mundo y otras 20-50 millones de personas con lesiones por traumatismos no mortales. Sin embargo, estos números son más preocupantes en términos de salud pública mundial, ya que es la primera causa de muerte en la población de 15-29 años, la segunda en el grupo de 5-14 años y la tercera en el de 30-44 años.

Es claro entonces que el mundo, en el campo de la salud, se encuentra en un proceso de transición epidemiológica en el cual las enfermedades transmisibles infectocontagiosas han ido cediendo posiciones en el ranking de mortalidad y morbilidad a las enfermedades no transmisibles, asociadas, en gran medida, a enfermedades cardíacas, cáncer, lesiones -entre ellas las causadas por el tránsito- y los trastornos mentales. Estas tienen como característica común permitir muchos años de vida, pero con una calidad de vida reducida. Así pues, han surgido estudios sobre la "Carga Global de Enfermedad" o conceptos como "Años de Vida Perdidos y Vividos con Discapacidad" (DALYs, Disability Adjusted Life Year), etc. Todos ellos se enfocan en conocer y mensurar el impacto que tienen las distintas enfermedades en la calidad de vida de las personas. ${ }^{2}$

En nuestro país, existen muchas instituciones que se ocupan de registrar datos relacionados con la siniestralidad vial y sus víctimas, por ejemplo, el Ministerio de Salud de la Nación (a través de la Encuesta Nacional de Factores de Riesgo o de la Dirección de Estadísticas e Información en Salud -DEIS-), la Dirección Nacional de Información Operacional y Mapa del Delito (DNIO) perteneciente al Ministerio de Seguridad de la Nación, la Dirección Nacional de Observatorio Vial (DNOV), la Dirección Nacional de Vialidad, entre otros. Todos estos actores participan de la concentración de los datos y conforman la Red Nacional de Estadística Vial, establecida por la Ley 26.363 y que creó, en 2008, la Agencia Nacional de Seguridad Vial (ANSV). En consecuencia, la Red Nacional de Estadística Vial de la ANSV es considerada la principal fuente de información para dimensionar la mortalidad causada por la siniestralidad vial en la Argentina. ${ }^{3,4}$

Según de la ANSV, en la Argentina, fallecieron 5611 personas como consecuencia de los siniestros viales en 2017 y más de 100.000 resultaron heridas. Por su parte, las muertes por causa externa -entre las cuales se incluyen las defunciones por lesiones de tránsito- representan la cuarta causa de muerte en el país, principalmente en personas de 15 a 34 años, según los datos de 2014 de la DEIS. De acuerdo con el Institute for Health Metrics and Evaluation (IHME), las muertes prematuras por lesiones de tránsito en la Argentina han pasado del sexto lugar en 2005 al cuarto en 2015, lo que pone de manifiesto el avance de esta problemática en el país y reconoce a los incidentes viales como un problema de salud pública de gran relevancia por la pérdida de vidas jóvenes y la carga de secuelas físicas y psicológicas que genera. ${ }^{2}$

La ANSV y todos los organismos que participan en este "Observatorio Vial" trabajan para alcanzar el registro sistemático de los siniestros viales y sus consecuencias en términos de morbimortalidad, procurando relevar el universo de hechos ocurridos en todo el territorio nacional, con el objetivo de generar información que contribuya a la toma de decisiones en la gestión y la coordinación de estrategias para el abordaje de esta problemática (prevención, educación, inversión en infraestructura, etc.).

En cuanto al tipo de lesiones traumatológicas y su evolución, originadas en los accidentes de tránsito, no tenemos un registro nacional que recopile datos específicos que nos permitan establecer la morbimortalidad en nuestra población, ni obtener información epidemiológica nacional segura que posteriormente se pueda aplicar a estrategias de atención protocolizada en el país, inversión hospitalaria eficiente $\mathrm{y}$, finalmente, publicaciones con datos propios que aumenten su validez científica.

En este sentido, en 2010, se creó el Comité de Morbimortalidad de la Asociación Argentina de Ortopedia y Traumatología, al comprender esta necesidad mundial de recopilar datos en relación con la morbimortalidad de las enfermedades no transmisibles y vinculadas a nuestra especialidad. En 2015, ha publicado una encuesta sobre las lesiones osteoarticulares ocurridas en siniestros viales. Esta encuesta está dirigida a todas las instituciones con servicio traumatológico de emergencia del país. 
El objetivo general de este informe es presentar los datos obtenidos desde 2017 hasta el primer semestre de 2020 , pero, más bien, con un objetivo específico: promover el registro sistemático de datos vinculados a las lesiones en nuestra especialidad producidas en el contexto de los siniestros viales, en virtud de la implicancia actual y futura de estas enfermedades en la salud pública.

En un primer informe (que se puede consultar en la página oficial de la AAOT), se publicaron los resultados de 155 encuestas acumuladas en el período 2015-2016. En este nuevo informe, se dan a conocer los resultados obtenidos de las 118 encuestas acumuladas entre enero de 2017 y el 30 de junio de 2020 . No fue posible comparar las muestras por período anual, debido a los pocos registros acumulados por año. Sin embargo, consideramos oportuno, al menos, compararlos como dos grupos de muestras. Algunas de las variables en estudio no podrán compararse, pues se han modificado con respecto a la encuesta original con el fin de agilizarla y ampliar la información con variables más específicas para nuestra práctica.

\section{RESULTADOS}

Con respecto al período 2015-2016, se observó un 15\% más de participación desde el ámbito público y un descenso del ámbito privado y de Obra Social (Tablas 1 y 2).

Tabla 1. Tipo de institución: distribución de frecuencia en el aporte de datos

\begin{tabular}{|l|c|c|}
\hline Tipo de institución & Frecuencia & Porcentaje \\
\hline Pública & 106 & $89,8 \%$ \\
\hline Privada & 9 & $7,6 \%$ \\
\hline Obra Social & 3 & $2,5 \%$ \\
\hline Total & 118 & $100 \%$ \\
\hline
\end{tabular}

Tabla 2. Instituciones participantes en el aporte de datos

\begin{tabular}{|l|c|c|}
\hline Instituciones & Frecuencia & Porcentaje \\
\hline Hospital "Carlos G. Durand” & 36 & $31,9 \%$ \\
\hline Hospital "Parmenio Piñero" & 11 & $9,7 \%$ \\
\hline Hospital "Francisco Santojanni” & 4 & $3,5 \%$ \\
\hline Hospital Británico & 5 & $4,4 \%$ \\
\hline Hospital C. San Martín & 24 & $21,2 \%$ \\
\hline HIGA “Prof. Dr. Rodolfo Rossi” & 4 & $3,5 \%$ \\
\hline Sanatorio Modelo & 3 & $2,7 \%$ \\
\hline HIGA “San José” & 5 & $4,4 \%$ \\
\hline Hospital Naval Puerto Belgrano & 2 & $1,8 \%$ \\
\hline Hospital Independencia & 12 & $10,6 \%$ \\
\hline Hospital Central de Mendoza & 7 & $6,2 \%$ \\
\hline Total & 113 & $100 \%$ \\
\hline
\end{tabular}


Las regiones representadas en las encuestas se detallan en la Tabla 3.

Tabla 3. Provincias: distribución de frecuencia en el aporte de datos

\begin{tabular}{|l|c|c|}
\hline Provincia participante & Frecuencia & Porcentaje \\
\hline Ciudad Autónoma de Buenos Aires & 51 & $43,2 \%$ \\
\hline Buenos Aires & 43 & $36,4 \%$ \\
\hline Santiago del Estero & 12 & $10,2 \%$ \\
\hline Mendoza & 7 & $5,9 \%$ \\
\hline No especificada & 5 & $4,2 \%$ \\
\hline Total & 118 & $100 \%$ \\
\hline
\end{tabular}

\section{Variables analizadas}

Sexo: los porcentajes fueron similares a los del informe previo, pero con un $2 \%$ más de mujeres (Tabla 4).

Tabla 4. Frecuencia de accidentados según el sexo

\begin{tabular}{|l|c|c|}
\hline Sexo & Frecuencia & Porcentaje \\
\hline Femenino & 29 & $24,6 \%$ \\
\hline Masculino & 89 & $75,4 \%$ \\
\hline No especificado & 1 & $0,8 \%$ \\
\hline Total & 118 & $100 \%$ \\
\hline
\end{tabular}

Edad: predominó el rango de 20 a 49 años, dato similar al del informe previo. Le siguió el rango de 50 a 70 años (pero, a diferencia del informe previo, sin ningún paciente mayor de 70 años) (Tabla 5).

Tabla 5. Frecuencia de accidentes según la edad

\begin{tabular}{|l|c|c|}
\hline Rango etario (años) & Frecuencia & Porcentaje \\
\hline$<20$ & 18 & $15,3 \%$ \\
\hline $20-49$ & 79 & $66,9 \%$ \\
\hline $50-70$ & 21 & $17,8 \%$ \\
\hline Total & 118 & $100 \%$ \\
\hline
\end{tabular}


Día de ingreso en el servicio de emergencia: en comparación con los resultados de informe anterior, el dato que se mantuvo constante fue el día jueves con el menor número de ingresos. Los datos de las encuestas de 2015-2016 sobre el horario del accidente, el tipo de transporte y el rol del lesionado involucrado en el siniestro no se incluyeron en las encuestas posteriores, por lo que no disponemos de datos comparativos (Tabla 6).

Tabla 6. Distribución de frecuencia según qué día de la semana ocurrió el accidente

\begin{tabular}{|l|c|c|}
\hline Día de la semana & Frecuencia & Porcentaje \\
\hline Lunes & 7 & $5,9 \%$ \\
\hline Martes & 15 & $12,7 \%$ \\
\hline Miércoles & 23 & $19,5 \%$ \\
\hline Jueves & 7 & $5,9 \%$ \\
\hline Viernes & 17 & $14,4 \%$ \\
\hline Sábado & 26 & $22,0 \%$ \\
\hline Domingo & 17 & $14,4 \%$ \\
\hline No especificado & 6 & $5,1 \%$ \\
\hline Total & 118 & $100 \%$ \\
\hline
\end{tabular}

Politraumatismo: no se comunicó este dato en el $72 \%$, por lo que no fue posible estimar la frecuencia de esta variable en la serie.

Traumatismo encefalocraneano: solo se informó en 87 casos. En las 31 encuestas restantes (26,3\%), no se indicó su ausencia, por lo que tampoco fue posible establecer la frecuencia en esta serie. Sin embargo, el $93 \%$ de los 87 casos informados con traumatismo encefalocraneano no tuvo pérdida del conocimiento (en el informe previo, la frecuencia fue del 78\%) (Tabla 7).

Tabla 7. Distribución de frecuencia del traumatismo encefalocraneano con pérdida de la conciencia o sin ella

\begin{tabular}{|l|c|c|}
\hline Traumatismo encefalocraneano & Frecuencia & Porcentaje \\
\hline Sin pérdida de la conciencia & 81 & $68,6 \%$ \\
\hline Con pérdida de la conciencia & 6 & $5,1 \%$ \\
\hline No especificado & 31 & $26,3 \%$ \\
\hline Total & 118 & $100 \%$ \\
\hline
\end{tabular}


Naturaleza de la lesión: se informaron 136 lesiones en 118 casos registrados: el 87,5\% tenía fracturas, este dato se mantuvo como la lesión más frecuente en ambos informes (2015-2016, 71,6\%) (Tabla 8).

Tabla 8. Distribución de frecuencia según la naturaleza de la lesión

\begin{tabular}{|l|c|c|}
\hline Naturaleza de la lesión & Frecuencia & Porcentaje \\
\hline Fractura & 119 & $87,5 \%$ \\
\hline Luxación & 4 & $2,9 \%$ \\
\hline Luxofractura & 5 & $3,7 \%$ \\
\hline Otras lesiones & 8 & $5,9 \%$ \\
\hline Total & 136 & $100 \%$ \\
\hline
\end{tabular}

Si bien no fue posible establecer una asociación entre las variables "Traumatismo encefalocraneano" y "Naturaleza de la lesión", debido al subregistro, las fracturas fueron la lesión que más se asoció con traumatismo encefalocraneano (Tabla 9).

Tabla 9. Asociación de traumatismo encefalocraneano y lesiones osteoarticulares: distribución de frecuencia

\begin{tabular}{|l|c|c|c|c|}
\hline \multirow{2}{*}{$\begin{array}{l}\text { Tipo de lesión } \\
\text { osteoarticular }\end{array}$} & \multicolumn{4}{|c|}{ Traumatismo encefalocraneano } \\
\cline { 2 - 5 } & $\begin{array}{c}\text { Sin pérdida de la } \\
\text { conciencia (\%) }\end{array}$ & $\begin{array}{c}\text { Con pérdida de la } \\
\text { conciencia (\%) }\end{array}$ & No especificado (\%) & Total (\%) \\
\hline Fractura & $70(59,3 \%)$ & $6(5,1 \%)$ & $26(22 \%)$ & $102(86,4 \%)$ \\
\hline Luxación & $1(0,8 \%)$ & $0 \%$ & $2(1,7 \%)$ & $3(2,5 \%)$ \\
\hline Luxofractura & $3(2,5 \%)$ & $0 \%$ & $2(1,7 \%)$ & $5(4,2 \%)$ \\
\hline Otras lesiones & $7(5,9 \%)$ & $0 \%$ & $1(0,8 \%)$ & $8(6,8 \%)$ \\
\hline Total & $81(68,6 \%)$ & $6(5,1 \%)$ & $31(26,3 \%)$ & $118(100 \%)$ \\
\hline
\end{tabular}

Localización de las lesiones (Tablas 10-12): En relación con el tipo de lesión, de las 115 especificadas, el porcentaje se mantuvo similar al del informe previo de 2015-2016, con un 72,2\% ( $\mathrm{n}=83$ ) de fracturas en el miembro inferior y un $22,6 \%(n=26)$ en el miembro superior. Las luxofracturas predominaron en el miembro superior $(6,9 \%)$, a diferencia de informe previo donde predominó el miembro inferior. Sin embargo, las luxaciones mantuvieron su predominio en el miembro inferior. Se detectó un aumento en la frecuencia en relación con las fracturas en el miembro inferior: pierna y rodilla $49,4 \%$ y $19,3 \%$, respectivamente, frente al $19,3 \%$ y $9,6 \%$ en el informe de 2015-2016; tobillo: 13,3\% frente a un 9,6\% en el informe previo. En cambio, sobre el fémur, la cadera y el pie, se observó un descenso de la frecuencia: $10,8 \%, 6 \%$ y 1,1\%, respectivamente, frente al 18,5\%, $11,9 \%$ y $3,7 \%$, respectivamente, en el informe anterior. 
Tabla 10. Distribución de frecuencia de lesiones según la región anatómica

\begin{tabular}{|l|c|c|}
\hline Lesiones según la región & Frecuencia & Porcentaje \\
\hline Raquis & 3 & $2,2 \%$ \\
\hline Pelvis & 3 & $2,2 \%$ \\
\hline Miembro inferior & 91 & $67,4 \%$ \\
\hline Miembro superior & 29 & $21,5 \%$ \\
\hline No especificada & 9 & $6,7 \%$ \\
\hline Total & $135^{*}$ & $100 \%$ \\
\hline
\end{tabular}

*En 7 casos (5,1\%), se informó asociación de lesiones en más de una región.

Tabla 11. Distribución de frecuencia de lesiones en el miembro inferior

\begin{tabular}{|l|c|c|}
\hline $\begin{array}{l}\text { Localización de la lesión en el miembro } \\
\text { inferior }\end{array}$ & Frecuencia & Porcentaje \\
\hline Acetábulo & 2 & $2,2 \%$ \\
\hline Cadera & 7 & $7,7 \%$ \\
\hline Fémur & 9 & $9,9 \%$ \\
\hline Pierna & 41 & $45,1 \%$ \\
\hline Rodilla & 19 & $20,9 \%$ \\
\hline Tobillo & 12 & $13,2 \%$ \\
\hline Pie & 1 & $1,1 \%$ \\
\hline Total & 91 & $100 \%$ \\
\hline
\end{tabular}

Tabla 12. Distribución de frecuencia de lesiones en el miembro superior

\begin{tabular}{|l|c|c|}
\hline $\begin{array}{l}\text { Localización de la lesión en el miembro } \\
\text { superior }\end{array}$ & Frecuencia & Porcentaje \\
\hline Cintura escapular & 7 & $24,1 \%$ \\
\hline Brazo & 5 & $17,2 \%$ \\
\hline Codo & 2 & $6,9 \%$ \\
\hline Antebrazo & 7 & $24,1 \%$ \\
\hline Muñeca & 7 & $24,1 \%$ \\
\hline Mano & 1 & $3,4 \%$ \\
\hline Total & 29 & $100 \%$ \\
\hline
\end{tabular}


En relación con las fracturas en el período 205-2016, se comunicó el mismo orden de frecuencia en el miembro superior, a excepción del codo que mantuvo una mayor frecuencia que el húmero. En la pelvis y el raquis, no se informó ninguna en 2015-2016 (Tablas 8, 13-15).

Tabla 13. Distribución de frecuencia del tipo de lesión según el sector del miembro inferior

\begin{tabular}{|l|c|c|c|c|c|}
\hline \multirow{2}{*}{$\begin{array}{l}\text { Sectores del } \\
\text { miembro Inferior }\end{array}$} & \multicolumn{5}{|c|}{ Tipo de lesión } \\
\cline { 2 - 6 } & Fractura & Luxofractura & Luxación & Otras lesiones & Total \\
\hline Cadera & 5 & 2 & 2 & 0 & $9(9,9 \%)$ \\
\hline Muslo & 9 & 0 & 0 & 0 & $9(9,9 \%)$ \\
\hline Rodilla & 16 & 0 & 2 & 1 & $19(20,9 \%)$ \\
\hline Pierna & 41 & 0 & 0 & 0 & $41(45 \%)$ \\
\hline Tobillo & 11 & 1 & 0 & 0 & $12(13,2 \%)$ \\
\hline Pie & 1 & 0 & 0 & 0 & $1(1,1 \%)$ \\
\hline Total & $83(91,2 \%)$ & $3(3,3 \%)$ & $4(4,4 \%)$ & $1(1,1 \%)$ & $91(100 \%)$ \\
\hline
\end{tabular}

Tabla 14. Distribución de frecuencia del tipo de lesión según el sector del miembro superior

\begin{tabular}{|l|c|c|c|c|c|}
\hline \multirow{2}{*}{$\begin{array}{l}\text { Sectores del } \\
\text { miembro superior }\end{array}$} & \multicolumn{4}{|c|}{ Tipo de lesión } & \\
\cline { 2 - 6 } & Fractura & Luxofractura & Luxación & Otras lesiones & Total \\
\hline Hombro & 6 & 0 & 0 & 1 & $7(24,1 \%)$ \\
\hline Brazo & 5 & 0 & 0 & 0 & $5(17,2 \%)$ \\
\hline Codo & 2 & 0 & 0 & 0 & $2(6,9 \%)$ \\
\hline Antebrazo & 6 & 0 & 0 & 0 & $6(20,7 \%)$ \\
\hline Muñeca & 6 & 2 & 0 & 0 & $8(27,5 \%)$ \\
\hline Mano & 1 & 0 & 0 & $1(3,4 \%)$ \\
\hline Total & $26(89,7 \%)$ & $2(6,9 \%)$ & $0 \%$ & $1(3,4 \%)$ & $29(100 \%)$ \\
\hline
\end{tabular}

Tabla 15. Distribución de frecuencia del tipo de lesión según el sector del eje axial

\begin{tabular}{|l|c|c|c|c|}
\hline Tipo de lesión en el eje axial & Anillo pélvico & Sacro & Vértebra (lumbar) & Total \\
\hline Fractura & 2 & 2 & 2 & $6(85,7 \%)$ \\
\hline Otras lesiones & 1 & 0 & 0 & $1(14,3 \%)$ \\
\hline Total & $3(42,8 \%)$ & $2(28,5 \%)$ & $2(28,5 \%)$ & $7(100 \%)$ \\
\hline
\end{tabular}


Condición de la lesión (abierta o cerrada): se destaca que predominó la lesión cerrada, al igual que en el período 2015-2016 (Tabla 16).

Tabla 16. Distribución de frecuencia de la naturaleza de la lesión según su condición

\begin{tabular}{|l|c|c|c|c|}
\hline \multirow{2}{*}{ Naturaleza de la lesión } & \multicolumn{4}{|c|}{ Condición de la lesión } \\
\cline { 2 - 5 } & Abierta & Cerrada & No especificada & Total \\
\hline Fractura & 35 & 48 & 36 & $119(87,5 \%)$ \\
\hline Luxación & 0 & 3 & 1 & $4(2,9 \%)$ \\
\hline Luxofractura & 3 & 2 & 0 & $5(3,7 \%)$ \\
\hline Otras lesiones & 1 & 1 & 6 & $8(5,9 \%)$ \\
\hline Total & $39(28,7 \%)$ & $54(39,7 \%)$ & $43(31,6 \%)$ & $136(100 \%)$ \\
\hline
\end{tabular}

Cuando se especificó una lesión osteoarticular y su condición en comparación con el período 2015-2016, la fractura abierta de pierna se ubicó en primer lugar, antes que el fémur. Las fracturas abiertas restantes fueron en el miembro superior, todas en el antebrazo o la muñeca, a diferencia del informe previo donde predominó ligeramente la fractura de húmero (Tabla 17).

Tabla 17. Distribución de frecuencia de las lesiones osteoarticulares en función de su condición, por sector anatómico

\begin{tabular}{|c|c|c|c|c|c|c|}
\hline Sector & $\begin{array}{c}\text { Fractura } \\
\text { abierta }\end{array}$ & $\begin{array}{c}\text { Fractura } \\
\text { cerrada }\end{array}$ & $\begin{array}{c}\text { Luxofractura } \\
\text { abierta }\end{array}$ & $\begin{array}{c}\text { Luxofractura } \\
\text { cerrada }\end{array}$ & $\begin{array}{l}\text { Luxación } \\
\text { cerrada }\end{array}$ & $\begin{array}{c}\text { Total \% } \\
\text { (n) }\end{array}$ \\
\hline Acetábulo & $0 \%$ & $1,1 \%$ & $0 \%$ & $0 \%$ & $0 \%$ & $1,1 \%(1)$ \\
\hline Cadera & $0 \%$ & $4,4 \%$ & $0 \%$ & $2,2 \%$ & $2,2 \%$ & $8,8 \%(8)$ \\
\hline Fémur & $4,4 \%$ & $4,4 \%$ & $0 \%$ & $0 \%$ & $0 \%$ & $8,8 \%(8)$ \\
\hline Rótula & $0 \%$ & $2,2 \%$ & $0 \%$ & $0 \%$ & $0 \%$ & $2,2 \%(2)$ \\
\hline Rodilla & $0 \%$ & $0 \%$ & $0 \%$ & $0 \%$ & $1,1 \%$ & $1,1 \%(1)$ \\
\hline Platillo tibial & $3,3 \%$ & $6,6 \%$ & $0 \%$ & $0 \%$ & $0 \%$ & $9,9 \%(9)$ \\
\hline Pierna & $2,2 \%$ & $6,6 \%$ & $0 \%$ & $0 \%$ & $0 \%$ & $30,8 \%(28)$ \\
\hline Tobillo & $2,2 \%$ & $3,3 \%$ & $1,1 \%$ & $0 \%$ & $0 \%$ & $6,6 \%(6)$ \\
\hline Metatarsiano & $0 \%$ & $1,1 \%$ & $0 \%$ & $0 \%$ & $0 \%$ & $1,1 \%(1)$ \\
\hline Clavícula & $0 \%$ & $4,4 \%$ & $0 \%$ & $0 \%$ & $0 \%$ & $4,4 \%(4)$ \\
\hline Húmero & $0 \%$ & $5,5 \%$ & $0 \%$ & $0 \%$ & $0 \%$ & $5,5 \%(5)$ \\
\hline Cúbito proximal & $0 \%$ & $1,1 \%$ & $0 \%$ & $0 \%$ & $0 \%$ & $1,1 \%$ \\
\hline Antebrazo & $1,1 \%$ & $3,3 \%$ & $1,1 \%$ & $0 \%$ & $0 \%$ & $5,5 \%(5)$ \\
\hline Muñeca & $2,2 \%$ & $0 \%$ & $1,1 \%$ & $0 \%$ & $0 \%$ & $3,3 \%(3)$ \\
\hline Falange & $0 \%$ & $1,1 \%$ & $0 \%$ & $0 \%$ & $0 \%$ & $1,1 \%(1)$ \\
\hline Pelvis & $0 \%$ & $1,1 \%$ & $0 \%$ & $0 \%$ & $0 \%$ & $1,1 \%(1)$ \\
\hline Sacro & $0 \%$ & $1,1 \%$ & $0 \%$ & $0 \%$ & $0 \%$ & $1,1 \%(1)$ \\
\hline Vértebra lumbar & $0 \%$ & $2,2 \%$ & $0 \%$ & $0 \%$ & $0 \%$ & $2,2 \%(2)$ \\
\hline No especificado & $1,1 \%$ & $3,3 \%$ & $0 \%$ & $0 \%$ & $0 \%$ & $4,4 \%$ \\
\hline Total & $38,5 \%(35)$ & $52,7 \%(48)$ & $3,3 \%(3)$ & $2,2 \%(2)$ & $3,3 \%(3)$ & $100 \%(91)$ \\
\hline
\end{tabular}


Solo en 32 casos se especificó la clasificación de Gustilo (Tabla 18).

Tabla 18. Distribución de frecuencia de las lesiones abiertas según la clasificación de Gustilo

\begin{tabular}{|c|c|c|c|c|c|c|}
\hline Tipo de lesión abierta & $\begin{array}{c}\text { Gustilo } \\
\text { I }\end{array}$ & $\begin{array}{c}\text { Gustilo } \\
\text { II }\end{array}$ & $\begin{array}{l}\text { Gustilo } \\
\text { IIIA }\end{array}$ & $\begin{array}{l}\text { Gustilo } \\
\text { IIIB }\end{array}$ & $\begin{array}{c}\text { No } \\
\text { especificada }\end{array}$ & Total \\
\hline Fractura & 6 & 16 & 4 & 3 & 6 & $35(92,1 \%)$ \\
\hline Luxofractura & 1 & 1 & 0 & 1 & 0 & $3(7,9 \%)$ \\
\hline Total & $7(18,4 \%)$ & $17(45 \%)$ & $4(10,5 \%)$ & $4(10,5 \%)$ & $6(15,8 \%)$ & $38(100 \%)$ \\
\hline
\end{tabular}

Lateralidad: (Tabla 19).

Tabla 19. Lateralidad de las lesiones: distribución de frecuencia en los casos

\begin{tabular}{|l|c|c|}
\hline Lateralidad de la lesión & Frecuencia & Porcentaje \\
\hline Derecha & 44 & $37,3 \%$ \\
\hline Izquierda & 55 & $46,6 \%$ \\
\hline Bilateral & 2 & $1,7 \%$ \\
\hline No especificada & 17 & $14,4 \%$ \\
\hline Total & 118 & $100 \%$ \\
\hline
\end{tabular}

Compromiso polisectorial: (Tabla 20).

Tabla 20. Fracturas en más de un sector: distribución de frecuencia en los casos

\begin{tabular}{|l|c|c|}
\hline Asociación de fracturas & Frecuencia & Porcentaje \\
\hline En miembro inferior & 9 & $56,3 \%$ \\
\hline En miembro superior & 0 & $0 \%$ \\
\hline En miembro inferior y superior & 4 & $25 \%$ \\
\hline Raquis & 1 & $6,3 \%$ \\
\hline Raquis + miembro inferior & 2 & $12,5 \%$ \\
\hline Total & 16 & $100 \%$ \\
\hline
\end{tabular}


Tipo de implante en relación con su fabricación: (Tabla 21).

Tabla 21. Tipo de implante utilizado: distribución de frecuencia según el origen de fabricación

\begin{tabular}{|l|c|c|}
\hline Origen del implante & Frecuencia & Porcentaje \\
\hline Importado & 7 & $5,9 \%$ \\
\hline Nacional & 38 & $32,2 \%$ \\
\hline No especificado & 73 & $61,9 \%$ \\
\hline Total & 118 & $100 \%$ \\
\hline
\end{tabular}

Tratamientos utilizados: En el $71 \%$ de los casos, no se especificó el tratamiento seleccionado, por lo que no se pudieron establecer datos confiables sobre la muestra. Sin embargo, cuando se especificó (29\%), el clavo endomedular fue el más usado (Tabla 22).

Tabla 22. Tipo de tratamiento: distribución de frecuencia en los casos

\begin{tabular}{|l|c|c|}
\hline Tipo de tratamiento quirúrgico & Frecuencia & Porcentaje \\
\hline Clavo endomedular & 14 & $11,3 \%$ \\
\hline Placa bloqueada & 9 & $7,3 \%$ \\
\hline Placa convencional & 3 & $2,4 \%$ \\
\hline Tutor externo & 5 & $4,0 \%$ \\
\hline Osteodesis & 5 & $4,0 \%$ \\
\hline No especificado & 88 & $71,0 \%$ \\
\hline Total & 124 & $100 \%$ \\
\hline
\end{tabular}

Complicaciones posquirúrgicas: no se especificaron en el 98,3\% de los casos, por lo que no se pudo establecer una frecuencia en la muestra (Tabla 23).

Tabla 23. Complicaciones posquirúrgicas: distribución de frecuencia en los casos

\begin{tabular}{|l|c|c|}
\hline Complicaciones posquirúrgicas & Frecuencia & Porcentaje \\
\hline Sí & 2 & $1,7 \%$ \\
\hline No especificado & 116 & $98,3 \%$ \\
\hline Total & 118 & $100 \%$ \\
\hline
\end{tabular}


Antecedentes patológicos: ante la escasa recolección de datos, no pudimos establecer la frecuencia de aparición de complicaciones ni la asociación de comorbilidades (Tabla 24).

Tabla 24. Antecedentes patológicos: distribución de frecuencia en los casos

\begin{tabular}{|l|c|c|}
\hline Antecedentes patológicos & Frecuencia & Porcentaje \\
\hline Diabetes & 3 & $2,5 \%$ \\
\hline Hipertensión arterial & 4 & $3,4 \%$ \\
\hline No presenta & 49 & $41,5 \%$ \\
\hline No especificado & 62 & $52,5 \%$ \\
\hline Total & 118 & $100 \%$ \\
\hline
\end{tabular}

\section{DISCUSIÓN}

De acuerdo con el informe del Observatorio Nacional Vial y el Observatorio de Seguridad Vial de la Ciudad Autónoma de Buenos Aires, los tipos de lesiones que originaron las discapacidades o incluso la muerte en los lesionados graves que ingresaron en el sistema público de salud se concentraron en las extremidades (40,3\%), y las lesiones espinales, en la cabeza y en las extremidades fueron las principales causas de discapacidad a largo plazo. ${ }^{2}$

En la bibliografía consultada, como la Encuesta Nacional de Siniestros Viales, está explícito que, en la Argentina, la mayor parte de los datos existentes proviene exclusivamente de registros administrativos. Asimismo, en dichos informes, se pone de manifiesto el concepto de que las encuestas por muestreo y los censos son las principales fuentes estadísticas que se utilizan para la elaboración de bases de datos y se sigue trabajando desde los organismos relacionados con esta área para aumentar la recolección, por ejemplo, a partir de 2016, se puso en marcha el Sistema Electrónico de Datos de Seguridad Vial (SIGISVI) que facilitará el entrecruzamiento de la información con otras bases de datos. ${ }^{4}$

Es claro que las estadísticas constituyen una herramienta fundamental para conocer y tratar problemas en una población. ${ }^{4}$ En la actualidad, los datos estadísticos sobre las enfermedades específicas de nuestra especialidad y, en particular, las provocadas en siniestros viales, no están disponibles desde ningún organismo, por lo que no contamos con datos epidemiológicos representativos y confiables nacionales ni regionales. En este sentido, este Comité continúa con su estrategia de recolectar información que nos aporte esos datos.

\section{CONCLUSIONES}

Es necesario generar datos estadísticos confiables, nacionales y regionales, sobre las lesiones traumáticas más prevalentes en nuestra especialidad, de modo que nos proporcionen información epidemiológica confiable. Consideramos necesario, en un futuro cercano, proveer y mantener un registro nacional que nos permita fundamentar nuestra práctica médica y científica sobre datos confiables y propios.

Para ello remarcamos la importancia de que se incorporen encuestas como las presentadas por este Comité, en cada Servicio como un instrumento de recolección de datos. Convocamos a renovar el compromiso de todas las instituciones a nivel nacional, nucleadas en esta Asociación, para el aporte de estos datos.

Conflicto de intereses: Los autores no declaran conflictos de intereses.

ORCID de M. N. Sierto: https://orcid.org/0000-0003-3330-0360 ORCID de G. Garabano: https://orcid.org/0000-0001-5936-0607 ORCID de A. Cubecino: https://orcid.org/0000-0002-8955-6595
ORCID de N. A. Robador: https://orcid.org/0000-0002-2683-6725 ORCID de H. Simesen de Bielke: https://orcid.org/0000-0003-2922-1440 ORCID de J. Olivetto: https://orcid.org/0000-0001-9445-0704 


\section{BIBLIOGRAFÍA}

1. Organización Mundial de la Salud, Departamento de Prevención de la Violencia y los Traumatismos y Discapacidad (VIP). Informe sobre la situación mundial de la seguridad vial: es hora de pasar a la acción, 2009. [Consulta: 6 de febrero, 2021]. Disponible en: https://www.who.int/violence_injury_prevention/road_safety_status/2009

2. Keller ME, Azar J, Liendro N, Jakovcevic A, Roldan A, Vidales, et al. Estimación de la carga global de enfermedad por siniestros viales. Ciudad Autónoma de Buenos Aires. Año 2017. Observatorio Nacional Vial y el Observatorio de Seguridad Vial de CABA. Diciembre 2018. [Consulta: 6 de febrero, 2021]. Disponible en: https://www.argentina.gob.ar/sites/default/files/ansv_observatoriovial_dalys_caba2.pdf

3. Ministerio de Transporte de la Nación. Marzo 2018. Situación de la seguridad vial en Argentina. Datos y análisis para un abordaje integral del problema. Información disponible hasta 2016. [Consulta: 6 de febrero, 2021].

Disponible en: https://www.argentina.gob.ar/sites/default/files/situacion_de_la_seguridad_vial_en_la_argentina_25.06.pdf

4. Núñez RA, Acquaviva MA, Chindemi M, Favelukes S, Aron Badin M, De Cesare MD, et al. Programa de Estudios sobre Siniestros Viales. Ciudad Autónoma de Buenos Aires: Ediciones SAIJ; 2018. [Consulta: 28 de agosto, 2020). Disponible en: https://www.argentina.gob.ar/sites/default/files/programa_de_estudios_sobre_siniestros_viales.pdf 